\title{
Turning noise into geologic information: The next big step?— A joint EAGE/SEG Forum
}

\author{
ERIC VERSCHUUR, Delft University of Technology
}

$I_{d}$ is often said that one geophysicist's noise is another's data, but today it seems that it has never been more the case. Features of seismic records that once were considered noise are now routinely used to aid in imaging the Earth's interior and in deriving rock properties. Sacrosanct noise such as surface waves is now inverted to derive near-surface properties. Multiple reflections, once the interpreter's bane, have long been estimated and removed but now are being used in the imaging process. Even seismic interference is now actively pursued as a source of new data in the guise of simultaneous-source acquisition. And then there is the emerging use of passive seismic data, which once would have been considered as pure noise.

This trend of turning noise into data, and therefore information, is a long-held tradition in geophysics, and it continues at a quick pace. From 11-14 November, a joint EAGE/ SEG forum was held in Lisbon, Portugal, to explore the state of the art of "noise as signal," inviting contributions from all areas of geophysics under this rubric. Almost 50 participants from a wide range of geographic locations and application areas gathered in the beautiful and historic Pestana Palace Hotel in Lisbon to talk about the use of noise in simultaneous-source shooting, passive seismic recording, imaging of multiples, and full-waveform inversion. Eighty percent of the participants were also presenters. Lively presentations took place between discussions. The idea of a forum, as opposed to a regular workshop, is to enhance open-minded discussions. For the forum, only short presentation abstracts were required and note-taking was kept to a minimum. Furthermore, to enhance interaction among participants, there was a predefined, randomized seating in the room that changed every day.

On the first technical day, 12 November, the main topic was passive seismic and seismic interferometry. Presentations centered on various applications of seismic interferometry to passive data, both at small scale (near-surface application) and large (earthquake seismology). Some interesting observations were the fact that using passive data for retrieving PP body waves via interferometry remains challenging. Usually, the passive data are overwhelmed by waves that travel more or less horizontally along the surface, rather than events coming from below. An exception to this may be only the microseismic events generated during production. However, this makes passive interferometry well suited to get information about the subsurface via surface waves. Various authors showed that with the interferometry of passive surface-wave energy, a good map of the near-surface (shear) wave velocity can be obtained, one that allows a model-driven approach for

Editor's note: Through cooperative efforts, this article is being published in both TLE and EAGE's First Break. near-surface correction, such as calculation of S-wave statics. This velocity inversion can be done by analyzing local surface wave properties or tomography methods based on arrivals across surface stations.

One interesting aspect of continuous active recording is that by low-pass filtering of the active measurements, we obtain perfect low-frequency passive measurements (assuming that the seismic source did not emit low frequencies) from which the velocities in the near surface can be obtained. As such, these freely obtained passive measurements are a great addition to active seismic measurements.

The second day of the forum was dedicated to simultaneous-source data, where the interference noise must be turned into signal using the right algorithms. For the marine case, it was shown that simultaneous-source acquisition allows undershooting of obstruction areas (such as around a platform) or the acquisition of ultralong offsets by using more than one source boat, where deblending algorithms could satisfactorily remove all the crosstalk. The latter becomes important for time-lapse studies because residual crosstalk noise should not interfere with the actual $4 \mathrm{D}$ signal. The latter turned out to be acceptable for a certain time-lapse project in the North Sea. However, during the discussion, it became clear that these results are still difficult to generalize into rules of thumb. It seems that everybody is comfortable with a simultaneoussource overlap of a factor of two or three, but larger factors are not yet proven to provide enough crosstalk suppression in the separation process. Furthermore, it also was shown that the ghost effect in marine measurements-normally assumed to provide no-data areas around the notch frequencies-could be fully recovered, thus providing full bandwidth output. This is another way of turning noise into signal.

In addition to organized simultaneous shooting, in practice we often face the problem of noise from a neighboring survey. A plea was made to share the coordinates and shooting times between surveys so that this crosstalk can be more easily predicted and subtracted.

For land data with typically lower signal-noise ratio values, more overlap is allowed. Furthermore, because of increased flexibility in land acquisition, new strategies can be developed, such as simultaneous shooting of surveys with different dedicated frequency bands and the accompanying equipment. As an example, the low-frequency survey can be shot with much coarser spatial sampling, allowing a quick coverage of the entire area.

It was noted that another advantage of continuous, simultaneous-source recording is that the length of shot records now becomes a processing parameter that can be chosen freely. This makes such measurement desirable also for the deep-imaging community because the Moho reflection will be detected by all such seismic-acquisition setups. 
The final day of the forum was dedicated to imaging and noise. First, the emphasis was on imaging diffractions separately from the specular reflections. Although everybody recognizes that diffractions are a genuine part of the physical subsurface response and, thereby, if properly handled by the current imaging algorithms, more information can be extracted by treating diffractions separately. By using dedicated separation algorithms, the image of the diffractions is proven to provide high-resolution information normally hidden in the seismic images. As proof, it turned out that the diffraction image showed more spatial detail than the output of the well-known coherency filters on the conventionally migrated cube. For this reason, this process provides a great way for interpreters to find small details such as faults, fractures, and channels.

In the last few years, great advancements have been made in the field of using all multiples, surface and internal, for imaging. Within this topic, we can distinguish using surface multiples in imaging by switching to different incident wavefields in the traditional migration process: Rather than using the original source wavelet as the downgoing signal, we must use the complete reflected wavefield at the surface as our incident field, after which all surface multiples are automatically migrated. However, this requires inversion-type imaging conditions to handle these complex wavefields and, in addition, define extra sparseness constraints on the image domain to suppress crosstalk. Some critical notes were made on the imaging of surface multiples because they may contain limited dip-angles compared to the imaged primaries. However, they compare favorable to primary images by considering their extended illumination area.

Bringing this one step further, broadband fullwaveform inversion can solve all modes of seismic data simultaneously (surface waves, primary reflections, multiples, refractions, and converted waves). The output will be a high-resolution velocity and density profile of the subsurface. However, it still requires a huge computational effort to solve this nonlinear inversion problem at the desired broadband frequency range.

Finally, a new imaging paradigm was presented via the so-called Marchenko inversion, in which the complete up- and downgoing wavefield, including the full multiple coda, can be reconstructed without knowledge of the velocity model via an autofocusing procedure.

In the near future, we will definitely see an increase in the use of multiples-both surface and internal multiples-in the imaging procedures, meaning that multiple-removal algorithms may become obsolete. However, primary and multiple separation algorithms may still play their role in a better-controlled imaging process.

As an overall conclusion to this forum, we can say that using what once was considered noise as signal now is becoming a well-established strategy and we definitely will see more benefits of these techniques in the future. Next, some acquisition tools may have to be redesigned to capture the wealth of information in our recordings. Finally, we hope to have a similar forum within a couple of years to monitor the progress in this field, and we are confident that the near future will bring us even more applications of turning noise into geologic information. THE

\section{Acknowledgments: The organizing committee for this forum} included Craig J. Beasley, WesternGeco; Panos G. Kelamis, Saudi Aramco; Dave Monk, Apache Corporation; Ramesh (Neelsh) Neelamani, ExxonMobil; Alexander Mihai Popovici, Z-Terra Inc.; Walter Rietveld, BP; and Eric Verschuur, Delft University of Technology.

\section{Corresponding author: D.J.Verschuur@tudelft.nl}

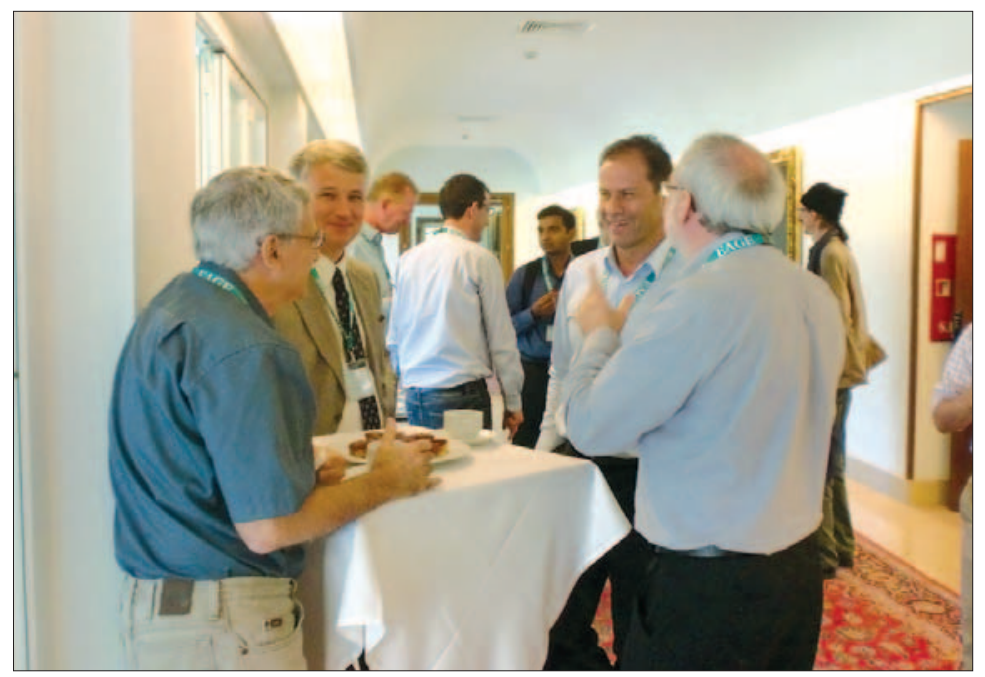

Forum participants continued the discussions during breaks. (Photo courtesy of Marjolein Verwey.)

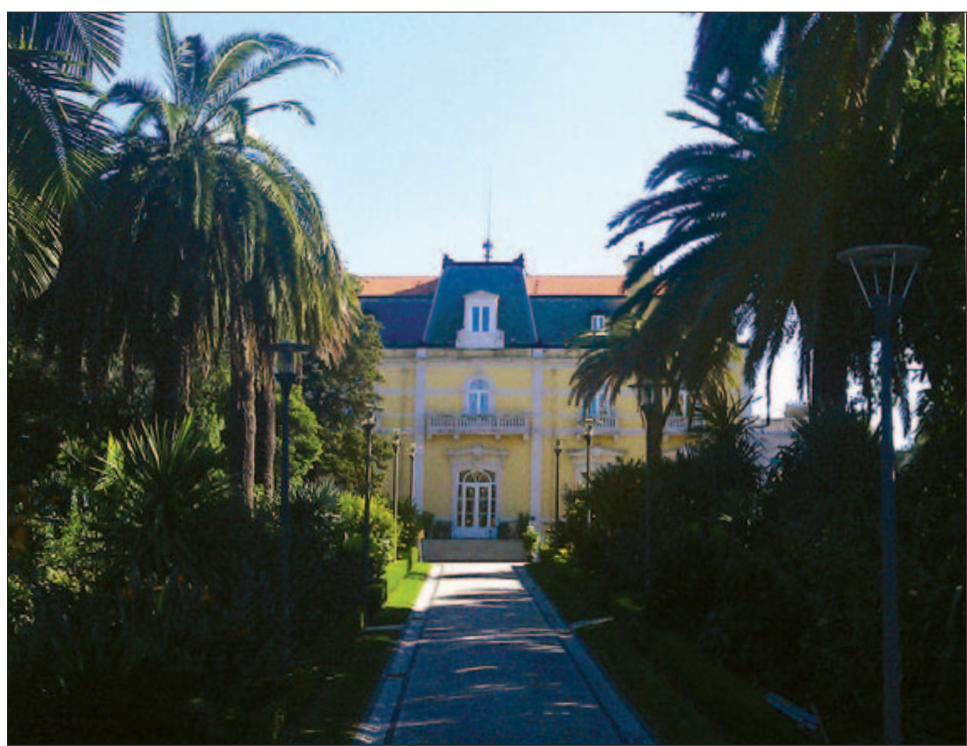

Pestana Palace, a national monument in Portugal, served as the meeting place for the EAGE/SEG Forum. (Photo courtesy of Craig Beasley.) 